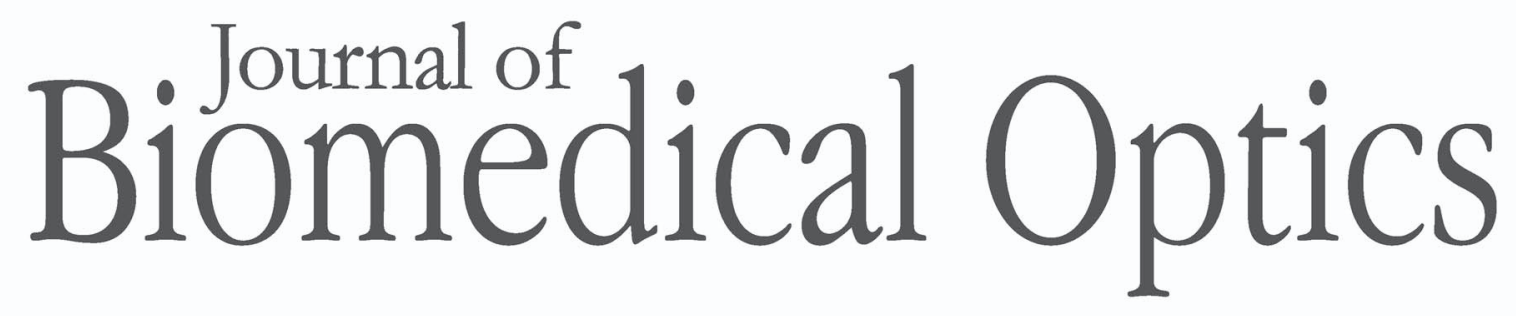

\title{
Raman spectroscopic characterization and differentiation of seminal plasma
}

Zufang Huang

Xiwen Chen

Yanping Chen

Jinhua Chen

Min Dou

Shangyuan Feng

Haishan Zeng

Rong Chen 


\section{Raman spectroscopic characterization and differentiation of seminal plasma}

\author{
Zufang Huang, ${ }^{a}$ Xiwen Chen, ${ }^{a}$ Yanping Chen, ${ }^{a}$ \\ Jinhua Chen, ${ }^{b}$ Min Dou, ${ }^{b}$ Shangyuan Feng, ${ }^{a}$ \\ Haishan Zeng, ${ }^{c}$ and Rong Chen ${ }^{a}$ \\ ${ }^{a}$ Fujian Normal University, Key Laboratory of Opto-Electronic \\ Science and Technology for Medicine of Ministry of Education, \\ Fujian Provincial Key Laboratory for Photonics Technology, \\ Fuzhou 350007, China \\ ${ }^{b}$ Fujian Provincial Hospital, Fuzhou 350001, China \\ ${ }^{\mathrm{c} B r i t i s h}$ Columbia Cancer Agency Research Centre, \\ Integrative Oncology Department, Imaging Unit, Vancouver, \\ BC V5Z 1L3, Canada
}

\begin{abstract}
Raman spectroscopy (RS) was applied for the analysis of seminal plasma in order to detect spectral parameters, which might be used for differentiating the normal and abnormal semen samples. Raman spectra of seminal plasma separated from normal and abnormal semen samples, showed a distinct difference in peak ratios between 1449 and $1418 \mathrm{~cm}^{-1}(P<0.05)$. More efficient alternative method of using principal component analysis-linear discriminate analysis based on Raman spectroscopic data yielded a diagnostic sensitivity of $73 \%$ and specificity of $82 \%$. The results suggest that RS combined with the multivariate analysis method has the potential for differentiating semen samples by examination of the corresponding seminal plasma. $\odot 2011$ Society of Photo-Optical Instrumentation Engineers (SPIE). [DOI: 10.1117/1.3650310]
\end{abstract}

Keywords: seminal plasma; Raman spectroscopy; multivariate analysis. Paper 11456LR received Aug. 24, 2011; revised manuscript received Sep. 17, 2011; accepted for publication Sep. 21, 2011; published online Oct. 26, 2011.

Clinically, semen analysis serves as an important criterion for evaluating the male reproductive capacity. Conventional semen analysis involving examination of the spermatozoa characteristics such as motility, forward progression, and concentration are routinely performed by laboratory operators, thus, user-induced errors due to manual operation are inevitable. Recently, studies focusing on automated analysis of semen samples have demonstrated the possibility with high precision and accuracy. ${ }^{1,2}$ However, these methods are still in controversy and not sufficient for clinical use. In addition, studies based on biochemical analysis or morphological characterization of spermatozoa were timeconsuming and labor-intensive, even with a risk of influencing the activity of spermatozoa when immobilizing the live spermatozoa for measurement. Knowing that semen consists of two parts, the cellular and noncellular parts (seminal plasma), semi-

Address all correspondence to: Rong Chen, Fujian Normal University, Key Laboratory of Optoelectronic Science and Technology for Medicine, Ministry of Education, Fuzhou, Fujian 0591 China, Tel: 01186059183165919 ; E-mail: chenr@fjnu.edu.cn. nal plasma plays a role as the soil for spermatozoa by providing a nutritive and protective medium during its fertilization, and evidence has shown that seminal plasma contains factors influencing male fertility. ${ }^{3,4}$ Thus, it is reasonable that analysis of seminal plasma will give an effective and objective reflection of the semen quality. Barcot et al. have presented encouraging results obtained from seminal plasma by using FTIR spectroscopy ${ }^{5}$ however, as the FTIR technique is limited by water interference, in their study the seminal plasma sample was dried before measurement, thus, it could induce physiological influence on seminal plasma; moreover, the broad-band spectral characteristic of FTIR would probably prevent some useful biomolecular information from being measured.

As a complementary technique to FTIR, Raman offers benefits that include minimal sample preparation, nondestructive analysis, and minimal interference from water. Raman spectroscopy (RS) in diagnostic aspects involving body fluids ${ }^{6-8}$ has been gaining importance in the last few years. However, so far RS has not been used to investigate and differentiate normal and abnormal semen samples based on the spectral characteristics of seminal plasma, and this is what motivated us to perform this work.

A set of 40 semen samples [18 normal and 22 abnormal, diagnosed according to World Health Organization (WHO) morphology criteria] were obtained, and each man produced a single sample by usual male fertility test routine after four to seven days of advised abstinence. After that, semen samples were incubated at $37^{\circ} \mathrm{C}$ for $20 \mathrm{~min}$ to achieve liquefaction and then an aliquot of semen sample were analyzed by computer-aided semen analysis. The remaining semen samples were freshly centrifuged for $10 \mathrm{~min}$ at $1000 \mathrm{~g}$ in eppendorf centrifuge and the seminal plasma was removed and separated from the semen. Prior to measurement, a small $10-\mu 1$ drop of seminal plasma was directly transferred with a pipette from the centrifuge tube onto an aluminum plate for subsequent experimentation.

A commercial Renishaw InVia confocal-Raman spectrometer coupled with a research-grade Leica microscope was used for all measurements. Through a $20 \times$ objective with a spectral resolution of $2 \mathrm{~cm}^{-1}, 785-\mathrm{nm}$ excitation light was focused on the sample. The laser power at the sample plane was $\sim 50 \mathrm{~mW}$ and the spot size of the excitation beam was $\sim 10 \mu \mathrm{m}$ wide by using the standard confocality mode. Raman signal detection was carried out using a Peltier cooled CCD camera with a 10 $\mathrm{s}$ integration time. Raw data obtained from samples were first treated using WiRE 2.0 software to remove any cosmic ray interference, then, fluorescence background was subtracted out using the program previously described by Zeng et al., ${ }^{9}$ and after that, each measured spectra were normalized to the integrated area under the curve range within 400 to $1800 \mathrm{~cm}^{-1}$.

Figure 1 shows reproducible mean spectra of seminal plasma obtained from 18 normal and 22 abnormal subjects. At first glance, peaks located at 527, 621, 716, 767, 956, 1004, 1063 to 1076,1321 to $1337,1418,1448$, and $1670 \mathrm{~cm}^{-1}$ can be clearly identified. The corresponding assignments of these peaks were shown in Table 1. When comparing the spectra of seminal plasma from normal and abnormal groups, we found a similar spectral profile, and the major differences are mostly of intensity variations, indicating that main differences exist in the content

1083-3668/2011/16(11)/110501/3/\$25.00 @ 2011 SPIE 


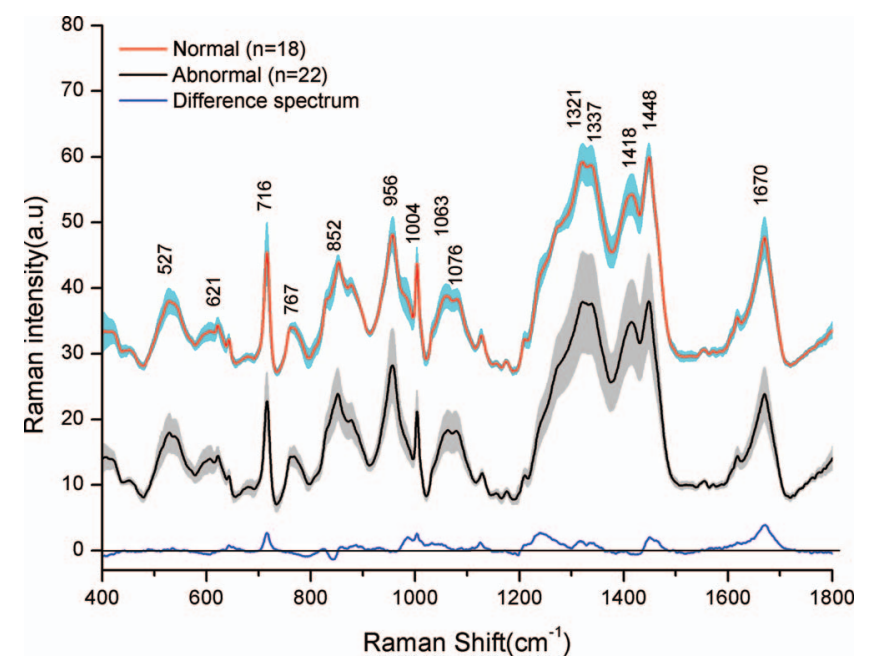

Fig. 1 Comparison of the mean spectrum for the normal seminal plasma (red solid curve, $n=18$ ) versus that of the abnormal (black solid curve, $n=22$ ). The shaded areas represent the standard deviations of the mean value. Also shown at the bottom is the difference spectrum.

change of certain compositions. An additional difference spectrum was shown at the bottom of Fig. 1, and the previously identified ${ }^{10}$ peaks at 716,1448 , and $1670 \mathrm{~cm}^{-1}$ are more intense in the normal group than in the abnormal group. In detail, the peak at $716 \mathrm{~cm}^{-1}$ is generally assigned to the C-N sym-

Table 1 Raman frequencies of measured range and their peak assignments.

\begin{tabular}{|c|c|}
\hline Raman shift $\left(\mathrm{cm}^{-1}\right)$ & Assignments \\
\hline 527 to 532 & Urea, skeletal deformation \\
\hline 621 & Ascorbic acid, cc aliphatic stretch \\
\hline 716 & $\begin{array}{l}\text { Nucleotide peak symmetric choline } \\
\text { stretch }\end{array}$ \\
\hline 767 & Symmetric ring breathing of tryptophan \\
\hline 851 to 852 & Ring breathing of tyrosine \\
\hline 956 & $\mathrm{PO}_{4}{ }^{3-}$ sym. stretching \\
\hline 1004 & $\begin{array}{l}\text { Phenylalanine, } v_{\mathrm{s}} \text { symmetric ring } \\
\text { breathing mode }\end{array}$ \\
\hline 1063 to 1076 & Protein, c-c skeletal stretching vibration \\
\hline 1321 & $\begin{array}{l}\text { Guanine, } \mathrm{CH}_{3} \mathrm{CH}_{2} \text { wagging nucleic } \\
\text { acids }\end{array}$ \\
\hline 1337 & Amide III, hydrated $\alpha$-helix $\delta(\mathrm{N}-\mathrm{H})$ \\
\hline 1418 & Lipids, $\alpha$-methylene $\mathrm{CH}_{2}$ scissoring band \\
\hline 1448 & Tryptophane, $\mathrm{CH}_{2}, \mathrm{CH}_{3}$ bending mode \\
\hline 1670 & Amide I, Backbone $\mathrm{C}=\mathrm{C}$ bond stretching \\
\hline
\end{tabular}

metric stretching vibration of choline, ${ }^{11-13}$ peak at $1448 \mathrm{~cm}^{-1}$ is contributing to $\mathrm{CH}_{2}, \mathrm{CH}_{3}$ bending mode in tryptophane, and $1670 \mathrm{~cm}^{-1}$ assigned to the $\mathrm{C}=\mathrm{C}$ bond stretching mode is attributed to polypeptide backbone of amide I. ${ }^{14}$ According to the peak assignments shown in Table 1, these results indicate that overall, relatively higher content of choline and spermine phosphate hexahydrate (SPH) was found in the normal group than that in the abnormal group. Interestingly, it was found that the peak of $1418 \mathrm{~cm}^{-1}$, which assigned to $\alpha$-methylene $\mathrm{CH}_{2}$ scissoring band of lipids, was previously described as a shoulder peak, ${ }^{15}$ but in our experiment, it appears as a more dominant peak. This may be due to the reason that when detecting the whole semen sample, spermatozoa produced a relatively larger signal at $1449 \mathrm{~cm}^{-1}$, which masked the peak of $1418 \mathrm{~cm}^{-1}$. By careful inspection of the obtained spectra, distinct spectral differences were seen at the 1449 and $1418 \mathrm{~cm}^{-1}$ peak positions. The average peak ratio of $1449 \mathrm{~cm}^{-1}$ versus $1418 \mathrm{~cm}^{-1}$ for the normal group $(1.13 \pm 0.05)$ is significantly different from that for the abnormal group $(1.26 \pm 0.15)$ (unpaired student's t-test, $P<0.05)$. That is, the ratio of Raman intensities at 1449 to $1418 \mathrm{~cm}^{-1}$ provided good differentiation between normal and abnormal groups.

However, to avoid spectral variations due to different detection spots in the same sample or different donors, a systematic and unbiased method for separating seminal plasma between normal and abnormal is quite essential. In our study, principal component analysis (PCA) coupled with linear discriminate analysis (LDA) was performed on the Raman spectra. First, PCA was applied to the Raman data for reducing complex data sets and extracting the key variables that account for the maximal variances in the multidimensional data set. After that, the principal component (PC) scores of the principal components were selected as variables for independent-sample $\mathrm{T}$ test. Two PCs (PC2 and PC4) were tested to be most diagnostically significant $(P<0.05)$ for discriminating normal and abnormal groups. To illustrate the use of PC scores for diagnostic classification, direct comparisons between normal and abnormal groups were

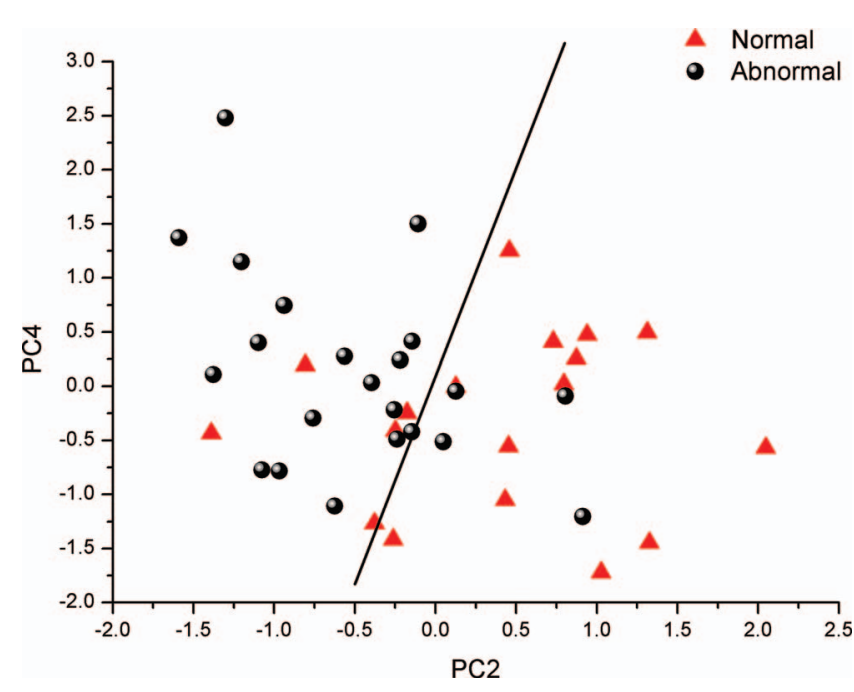

Fig. 2 Scatter plots of the second principal component (PC2) versus the fourth principal component (PC4) for normal group (triangle) versus abnormal group (solid sphere). The solid line (PC4 $=3.85 \mathrm{PC} 2$ $+0.093)$. 


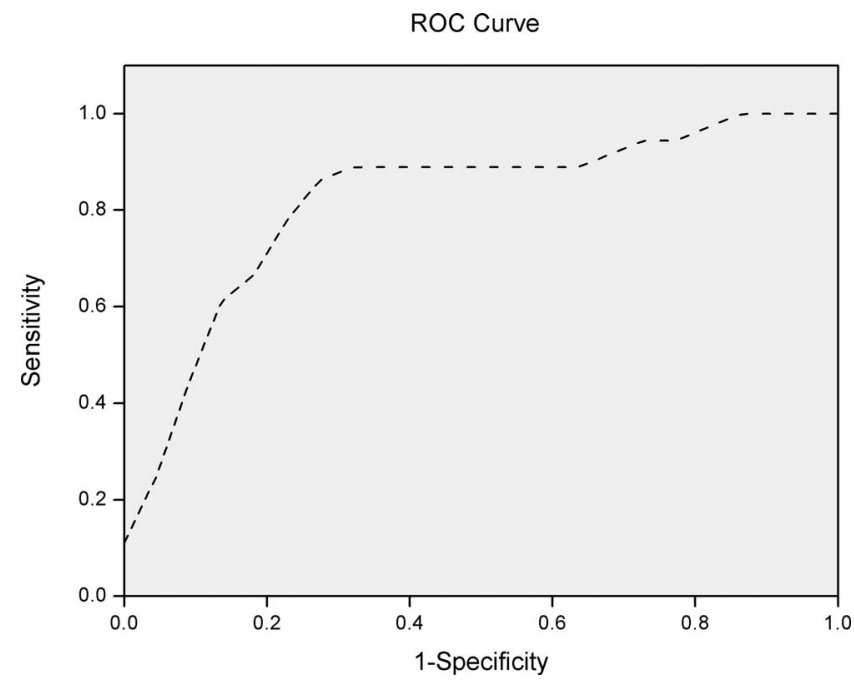

Fig. 3 ROC curve of the discrimination result by using PCA-LDA-based spectral classification with leave-one-out, cross-validation method. The integrated areas under the ROC curve are 0.823 .

presented in Fig. 2 by showing the scatter plot of the second PC versus the fourth PC. In order to incorporate all significant spectral features, LDA was used to generate diagnostic algorithms using the two most significant PCs (PC2 and PC4). In addition, for the validation of the PCA-LDA model, the leave-one-out cross-validation procedure was performed. ${ }^{16}$ The classification results showed sensitivity of $73 \%$ and specificity of $82 \%$. To further evaluate the performance of the PCA-LDA-based diagnostic algorithm for seminal plasma differentiation, the receive operator characteristic (ROC) curve, which was a comparison of two operating characteristics (true and false positive rates) as the criterion changes, was generated at different threshold levels (shown in Fig. 3). The area under the ROC curve was 0.823 showing that the Raman spectra of seminal plasma can be used for differentiation with high sensitivity and specificity.

In summary, our results suggest that it is feasible to use RS to characterize the seminal plasma. Specific spectral features and PCA-LDA analysis methods can be used to detect and differentiate semen samples by rapid and simple examination of the corresponding seminal plasma obtained from the normal and abnormal subjects. We envision the potential of using RS combined with multivariate statistical analysis as a new diagnostic method in semen quality assessment.

\section{Acknowledgments}

This work was supported by the National Natural Science Foundation of China (Grant Nos. 61178090, 81101110, and
11104030) and the Science and Technology Project of Fujian Province (Grant Nos. 2010J05130 and 2009J01276).

\section{References}

1. A. Agarwal and R. K. Sharma, "Automation is the key to standardized semen analysis using the automated SQA-V sperm quality analyzer," Fertil. Steril. 87(1), 156-162 (2007).

2. F. Ferrara, R. Daverio, G. Mazzini, P. Bonini, and G. Banfi, "Automation of human sperm cell analysis by flow cytometry," Clin. Chem. 43(5), 801-807 (1997).

3. E. P. Diamandis, W. P. Arnett, G. Foussias, H. Pappas, S. Ghandi, D. N. Melegos, B. Mullen, H. Yu, J. Srigley, and K. Jarvi, "Seminal plasma biochemical markers and their association with semen analysis findings," Urology 53(3), 596-603 (1999).

4. M. Kurpisz, R. Miesel, D. Sanocka, and P. Jedrzejczak, "Seminal plasma can be a predictive factor for male infertility," Hum. Reprod. 11(6), 1223-1226 (1996).

5. O. Barcot, M. Balarin, O. Gamulin, D. Jezek, P. Romac, and J. BrnjasKraljević, "Investigation of spermatozoa and seminal plasma by Fourier transform infrared spectroscopy," Appl. Spectrosc. 61(3), 309-313 (2007).

6. K. A. Esmonde-White, G. S. Mandair, F. Raaii, J. A. Jacobson, B. S. Miller, A. G. Urquhart, B. J. Roessler, and M. D. Morris, "Raman spectroscopy of synovial fluid as a tool for diagnosing osteoarthritis," $J$. Biomed. Opt. 14(3), 034013 (2009).

7. K. Virkler and I. K. Lednev, "Forensic body fluid identification: the Raman spectroscopic signature of saliva," Analyst 135(3), 512-517 (2010).

8. S. Feng, R. Chen, J. Lin, J. Pan, G. Chen, Y. Li, M. Cheng, Z. Huang, J. Chen, and H. Zeng, "Nasopharyngeal cancer detection based on blood plasma surface-enhanced Raman spectroscopy and multivariate analysis," Biosens. Bioelectron. 25(11), 2414-2419 (2010).

9. J. Zhao, H. Lui, D. I. McLean, and H. Zeng, "Automated autofluorescence background subtraction algorithm for biomedical Raman spectroscopy," Appl. Spectrosc. 61(11), 1225-1232 (2007).

10. K. Virkler and I. K. Lednev, "Raman spectroscopic signature of semen and its potential application to forensic body fluid identification," Forensic Sci. Int. 193(1-3), 56-62 (2009).

11. J. T. Edsall, "Raman spectra of amino acids and related compounds. VI. Sarcosine, ethanolamine, choline, betaine and betaine derivatives," J. Am. Chem. Soc. 65(9), 1767-1770 (1943).

12. Y. Koyama, S. Toda, and Y. Kyogoku, "Raman spectra and conformation of the glycerophosphorylcholine headgroup," Chem. Phys. Lipids 19(1), 74-92 (1977).

13. R. C. Spiker, Jr. and I. W. Levin, "Raman spectra and vibrational assignments for dipalmitoyl phosphatidylcholine and structurally related molecules," Biochim. Biophys. Acta. 388(3), 361-373 (1975).

14. J. D. Gelder, K. D. Gussem, P. Vandenabeele, and L. Moens, "Reference database of Raman spectra of biological molecules,"J. Raman Spectrosc. 38(9), 1133-1147 (2007).

15. K. Meister, D. A. Schmidt, E. Bründermann, and M. Havenith, "Confocal Raman microspectroscopy as an analytical tool to assess the mitochondrial status in human spermatozoa," Analyst 135(6), 1370-1374 (2010).

16. S. K. Teh, W. Zheng, K. Y. Ho, M. Teh, K. G. Yeoh, and Z. Huang, "Near-infrared Raman spectroscopy for early diagnosis and typing of adenocarcinoma in the stomach," Br. J. Surg. 97(4), 550-557 (2010). 